\title{
SINGULAR INTEGRALS WITH EXPONENTIAL WEIGHTS
}

\author{
E. PRESTINI
}

(Communicated by J. Marshall Ash )

Abstract. We study the operators

$$
\bar{V} f(t)=\frac{1}{w(t)} V(f(r) w(r))(t)
$$

where $V$ is the Hardy-Littlewood maximal function, the Hilbert transform or Carleson operator.

Under suitable conditions on the weight $w(t)$ of exponential type, we prove boundedness of $\bar{V}$ from $L^{p}$ spaces, defined on $[1,+\infty)$ with respect to the measure $w^{2}(t) d t$, to $L^{p}+L^{2}, 1<p \leq 2$, with the same density measure. These operators, that arise in questions of harmonic analysis on noncompact symmetric spaces, are bounded from $L^{p}$ to $L^{p}, 1<p<\infty$, if and only if $p=2$.

The study of convergence properties of inverse spherical transforms of radial functions on noncompact symmetric spaces $[1],[6],[7]$ requires estimates on singular integrals with exponential weights, as defined below, due to the exponential growth at infinity of the radial part $D(t) d t$ of the measure, where

$$
D(t)=(S h t)^{p}(S h 2 t)^{q},
$$

with $p$ and $q$ suitable nonnegative integers that depend upon the geometry of the symmetric space.

In what follows we define a class of functions $w(t)$, that include exponentials, and prove boundedness of the operators $\frac{1}{w(t)} V(f(r) w(r))(t)$ from $L^{p}\left(w^{2}(t) d t\right)$ to $L^{p}\left(w^{2}(t) d t\right)+L^{2}\left(w^{2}(t) d t\right), 1<p \leq 2$, where $V$ is the classical Hardy-Littlewood maximal function, the maximal Hilbert transform or the maximal Carleson operator. Boundedness from $L^{p}$ to $L^{p}, 1<p<\infty$, w.r. to the measure $w^{2}(t) d t$, holds for $p=2$ only.

Theorem 1. We denote by $L^{p}=L^{p}\left([1,+\infty), w^{2}(t) d t\right)$ the space of functions defined on $[1,+\infty)$ that are $L^{p}$ with respect to the density measure $w^{2}(t) d t$.

Received by the editors October 7, 1994

1991 Mathematics Subject Classification. Primary 42A50; Secondary 43A80. 
Let $\bar{V}$ be one of the following operators:

$$
\begin{aligned}
& \bar{M} f(t)=\frac{1}{w(t)} \sup _{\sigma>0} \frac{1}{2 \sigma} \int_{t-\sigma}^{t+\sigma}|f(r)| w(r) d r \\
& \bar{H} f(t)=\frac{1}{w(t)} \int_{1}^{\infty} \frac{f(r)}{t-r} w(r) d r \\
& \bar{C} f(t)=\frac{1}{w(t)} \sup _{R>1}\left|\int_{1}^{\infty} \frac{e^{i R(t-r)}}{t-r} f(r) w(r) d r\right| .
\end{aligned}
$$

Then $\bar{V}$ is a bounded operator from $L^{p}$ to $L^{p}+L^{2}, 1<p \leq 2$, provided that

i) $w(t) \geq a>0$ for all $t \in[1,+\infty)$;

ii) $w(t)$ satisfies the following "doubling condition"

$$
c_{1} w(k) \leq w(t) \leq c_{2} w(k) \quad \text { for all } t \in[k-1, k+2],
$$

where $k$ denotes any positive integer and the constants $c_{i}=c_{i}(w), i=1,2$, are positive and independent of $k$.

Proof. Let us observe that

$$
\bar{V} f(t)=\frac{1}{w(t)} V(f(r) w(r))(t)
$$

where $V$ denotes respectively the classical Hardy-Littlewood maximal function, the Hilbert transform and Carleson operators [3], [4], [8].

Due to the $L^{2}(\mathbb{R}, d r)$ boundedness of these classical operators the theorem in the case $p=2$ is trivial, for

$$
\begin{aligned}
\|\bar{V} f\|_{2}^{2} & =\int|\bar{V} f(t)|^{2} w^{2}(t) d t=\int|V(f(r) w(r))(t)|^{2} d t \\
& \leq \int|f(r)|^{2} w^{2}(r) d r=\|f\|_{2}^{2} .
\end{aligned}
$$

Next we decompose the operators as follows. For every fixed $k=1,2,3, \ldots$ we write

$$
\begin{aligned}
& g_{k}(r)=f(r) w(r) \chi_{[k, k+1)}(r), \\
& \varphi_{k}(t)=\chi_{[k-1, k+2)}(t), \\
& \varphi_{k}(t)+\psi_{k}(t)=1 \quad \text { for every } t \geq 1
\end{aligned}
$$

where $\chi_{E}$ denotes the characteristic function of the set $E$.

Suppose for instance $\bar{V}=\bar{M}$. Then observe that

$$
\frac{1}{2 \sigma} \int_{t-\sigma}^{t+\sigma}|f(r)| w(r) d r=\sum_{k=1}^{\infty}\left(\frac{1}{2 \sigma} \int_{t-\sigma}^{t+\sigma}\left|g_{k}(r)\right| d r\right)\left[\varphi_{k}(t)+\psi_{k}(t)\right]
$$

and

$$
\begin{aligned}
\bar{M} f(t) & \leq \frac{1}{w(t)} \sup _{\sigma} \sum_{k=1}^{\infty}\left(\frac{1}{2 \sigma} \int_{t-\sigma}^{t+\sigma}\left|g_{k}(r)\right| d r\right) \varphi_{k}(t) \\
& +\frac{1}{w(t)} \sup _{\sigma} \sum_{k=1}^{\infty}\left(\frac{1}{2 \sigma} \int_{t-\sigma}^{t+\sigma}\left|g_{k}(r)\right| d r\right) \psi_{k}(t) .
\end{aligned}
$$


We adopt a similar decomposition if $\bar{V}=\bar{H}$ or $\bar{C}$.

We shall prove that the first term on the right hand side of (2) maps boundedly $L^{p}$ into $L^{p}$, while the second one maps boundedly $L^{p}$ into $L^{2}, 1<p \leq 2$. The theorem then follows.

We start with the first term which is dominated by $\frac{1}{w(t)} \sum_{k} V g_{k}(t) \varphi_{k}(t)$. Since the $\varphi_{k}$ 's have essentially disjoint supports and $V$ is bounded on $L^{p}(\mathbb{R}, d r), 1<p<\infty$, we have

$$
\begin{aligned}
& \left\|\frac{1}{w(t)} \sum_{k=1}^{\infty} V g_{k}(t) \varphi_{k}(t)\right\|_{p}^{p} \\
& \cong \sum_{k} w^{-p}(k) \int_{k-1}^{k+2}\left|V g_{k}(t)\right|^{p} w^{2}(t) d t \leq c \sum_{k} w^{2-p}(k) \int_{1}^{\infty}\left|V g_{k}(t)\right|^{p} d t \\
& \leq c \sum_{k=1}^{\infty} w^{2-p}(k) \int_{1}^{\infty}\left|g_{k}(t)\right|^{p} d t=c \sum_{k=1}^{\infty} w^{2-p}(k) \int_{k}^{k+1}|f(r)|^{p} w^{p}(r) d r \\
& \cong \sum_{k=1}^{\infty} \int_{k}^{k+1}|f(r)|^{p} w^{2}(r) d r=c|| f \|_{p}^{p} .
\end{aligned}
$$

So we proved

$$
\left\|\frac{1}{w(t)} \sum_{k=1}^{\infty} V g_{k}(t) \varphi_{k}(t)\right\|_{p} \leq c_{p, w}\|f\|_{p}, \quad 1<p<\infty
$$

Next we deal with the second term on the right hand side of (2).

Suppose again $\bar{V}=\bar{M}$. Then from (1) we know that

$$
\frac{1}{w(t)} \sup _{\sigma} \sum_{k=1}^{\infty}\left(\frac{1}{2 \sigma} \int_{t-\sigma}^{t+\sigma}\left|g_{k}(r)\right| d r\right) \psi_{k}(t) \leq \bar{M} f+\frac{1}{w(t)} \sum_{k=1}^{\infty} V g_{k}(t) \varphi_{k}(t)
$$

is also bounded from $L^{2}$ to itself, due to the $L^{2}$ boundedness of $\bar{M} f$ and the estimate in (3) in the case $p=2$.

By standard interpolation techniques to prove boundedness of $\bar{M}$ from $L^{p}$ to $L^{2}$ it is then sufficient to show a restricted $\left(L^{p}, L^{2}\right)$ estimate, $1<p<2$.

Similarly for $\bar{H}$ and $\bar{C}$.

Now let $f=\chi_{E}, \quad E \subset[1,+\infty)$ any measurable set. In the case $\left\|\chi_{E}\right\|_{2} \geq 1$ the desired estimate is trivial and it is based on the $L^{2}$ boundedness of the operator we are dealing with, already established and the fact that $\left\|\chi_{E}\right\|_{2} \leq\left\|\chi_{E}\right\|_{p}, \quad 1<p \leq$ 2 .

If instead $\left\|\chi_{E}\right\|_{2}<1$, then we observe that for $t$ in the support of $\psi_{k}$

$$
M g_{k}(t)=\sup _{\sigma>0}\left(\frac{1}{2 \sigma} \int_{t-\sigma}^{t+\sigma}\left|g_{k}(r)\right| d r\right) \leq \frac{2}{|t-k|} \int_{k}^{k+1} \chi_{E}(r) w(r) d r .
$$

Indeed it has to be $\sigma \geq \frac{\text { dist }(t, k)}{2}$ to have a nonzero integral under the supremum. Therefore $\frac{1}{w(t)} \sum_{k} M g_{k}(t) \psi_{k}(t) \leq \frac{2}{w(t)} \frac{w(k)}{|t-k|}\left|E_{k}\right| \psi_{k}(t)$, when $\left|E_{k}\right|$ denotes the Eu- 
clidean measure of $E \cap[k, k+1)$. Then by i)

$$
\begin{aligned}
& \left\|\frac{1}{w(t)} \sum_{k} M g_{k}(t) \psi_{k}(t)\right\|_{2} \leq \sum_{k}\left\|\frac{1}{w(t)} \sum_{k} M g_{k}(t) \psi_{k}(t)\right\|_{2} \\
& \leq \sum_{k} w(k)\left|E_{k}\right|\left(\int \frac{1}{(t-k)^{2}} \psi_{k}(t) d t\right)^{\frac{1}{2}} \\
& \leq c \sum_{k} w(k)\left|E_{k}\right| \leq c_{w} \sum_{k} w^{2}(k)\left|E_{k}\right| \\
& \leq c_{w} \sum_{k}\left(\int_{k}^{k+1} \chi_{E}(r) d r\right) w^{2}(k) \cong c_{w} \sum_{k} \int_{k}^{k+1} \chi_{E}(r) w^{2}(r) d r \\
& =c_{w} \int_{1}^{\infty} \chi_{E}(r) w^{2}(r) d r \leq c_{w}\left\|\chi_{E}\right\|_{p} .
\end{aligned}
$$

In case $\bar{V}=\bar{H}$ or $\bar{C}$ the estimate in (4) can be obtained by taking the absolute value inside the sign of integral.

The theorem is then proved.

\section{Corollary. Let}

$$
H^{*} f(t)=\frac{1}{w(t)} \sup _{\varepsilon>0}\left|\int_{\substack{1 \\|t-r|>\varepsilon}}^{\infty} \frac{f(r)}{t-r} w(r) d r\right|
$$

and

$$
C^{*} f(t)=\frac{1}{w(t)} \sup _{\substack{R>1 \\ \varepsilon>0}}\left|\int_{\substack{1 \\|t-r|>\varepsilon}}^{\infty} \frac{e^{i R(t-r)}}{t-r} f(r) w(r) d r\right| .
$$

Under the same assumptions of Theorem $1, H^{*}$ and $C^{*}$ map boundedly $L^{p}$ into $L^{p}+L^{2}, \quad 1<p \leq 2$.

Proof. The Corollary follows at once from Theorem 1 and the estimate ([9], [5])

$$
H^{*} f(t) \leq \frac{1}{w(t)}[M(f(r) w(r))(t)+M H(f(r) w(r))(t)]
$$

and

$$
C^{*} f(t) \leq \frac{1}{w(t)}[M(f(r) w(r))(t)+M C(f(r) w(r))(t)]
$$

Theorem 2. Let $\bar{V}$ be as in Theorem 1. Then $\bar{V}$ is a bounded operator from $L^{p}$ to $L^{p}$ if and only if $p=2$.

Proof. We just have to prove unboundedness for $p \neq 2$. Suppose $1<p<2$ and take $f(r)=\chi_{[1,2]}(r), w(t)=e^{t}$. Then

$$
\bar{H} f(t) \cong \frac{1}{t e^{t}}, \text { for } t>>1
$$


From this the unboundedness of $\bar{H}$ follows. Now suppose $p>2$ and assume by contradiction that $\bar{H} f$ is a bounded operators from $L^{p}$ to the space of tempered distributions. Then by duality $\bar{H} f: C_{c}^{\infty} \rightarrow L^{p^{\prime}}, \quad p^{\prime}<2$, is a bounded operator. This is false as the preceding counterexample (slightly modified to have $f \in C^{\infty}$ ) shows.

Finally we observe that $w(t)=t^{\alpha} e^{\beta t}$ with $\alpha \in \mathbb{R}$ and $\beta>0$ satisfies the conditions of Theorem 1. In the case $\bar{V}=\bar{C}, \alpha=0$ and $\beta=\frac{p+2 q}{2}$ (more precisely $w(t)=\sqrt{D(t)}$, Theorem 1 has been used to obtain sharp results on almost everywhere convergence of inverse pherical transforms on noncompact symmetric spaces [7]. In [2] estimates in the context of Lorentz spaces have been obtained for $\bar{V}=\bar{H}$ in the case $\alpha=0$ and $\beta=1$.

\section{REFERENCES}

1. Colzani L., Crespi A., Travaglini G., Vignati M., Equiconvergence theorems for Fourier-Bessel expansions with applications to the harmonic analysis of radial functions in euclidean and non euclidean spaces, Trans. Amer. Math. Soc. 338 (1993), 43-55. MR 93j:42009

2. Colzani L., Vignati M., Hilbert transform with exponential weights, Proc. Amer. Math. Soc. 114, n.2 (1992), 451-457. MR 92e:44004

3. Carleson L., On convergence and growth of partial sums of Fourier series, Acta Math. 116 (1966), 135-157. MR 33:7774

4. Hunt R., On the convergence of Fourier series, Orthogonal Expansions and Continuous Analogues, Proc. Conference Edwardsville (Illinois 1967, SIU Press 1968), 235-255. MR 38:6296

5. Hunt R., Young W.S., A weighted norm inequality for Fourier series, Bull. Amer. Math. Soc. 80 (1974), 274-277. MR 49:3419

6. Meaney C., Prestini E., Almost everywhere convergence of inverse spherical transform on $S L(2, \mathbb{R})$, Ark. Mat. 32 (1994), 195-211. MR 95c:22015

7. Meaney C., Prestini E., Almost everywhere convergence of inverse spherical transforms on noncompact symmetric spaces, J. Funct. Anal. (to appear).

8. Sjolin P., Convergence almost everywhere of certain singular integrals and multiple Fourier series, Ark. Math. 9 (1971), 65-90. MR 49:998

9. Stein E.M., Singular Integrals and Differentiability Properties of Functions, Princeton Univ. Press (Princeton N.J. 1970). MR 44:7280

10. Bergh J., Löfström J., Interpolation Spaces, Springer-Verlag (Berlin 1976). MR 58:2349

Department of Mathematics, University of Rome, Tor Vergata, 00133 Rome, Italy

E-mail address: prestini@mat.utovrm.it 